Rose, H. S., \& Ludwig, T. D. (2009). Swimming Pool Hygiene: Self-Monitoring, Task Clarification, and Performance Feedback Increase Lifeguard Cleaning Behaviors. Journal of Organizational Behavior Management, 29(1), 69-79. doi:10.1080/01608060802660157 Version off record published by Published by Routledge / Taylor \& Francis and is available online at: http://www.informaworld.com/ (ISSN: 01608061)

\title{
Swimming Pool Hygiene: Self-Monitoring, Task Clarification, and Performance Feedback Increase Lifeguard Cleaning Behaviors
}

\author{
Henry M. S. Rose and Timothy D. Ludwig
}

\begin{abstract}
The effects of task clarification, self-monitoring, and performance feedback on cleaning behaviors of 9 lifeguards in 3 performance areas (vacuuming, lobby tidying, and pool deck maintenance) were investigated using an ABA reversal design at a county swim complex. A specific task in each performance area was used as a behavioral control. Following a task clarification meeting, the percentage of closing tasks completed each night was self-monitored through ratings by lifeguards and managers. Researchers conducted independent ratings of these completed tasks after the staff had left the building. Feedback data were posted daily using line graphs that displayed the percentage of tasks completed correctly from both self-report and researchers' data. Overall performance increased from an average of $45.1 \%$ correct behaviors during baseline to an average of $76.9 \%$ during intervention then reversed to baseline during follow-up to an average performance of $45.05 \%$.
\end{abstract}

\begin{abstract}
ARTICLE
Lifeguards who work intermittently may forget the detailed expectations required by the job. Ensuring that physical objects such as patrons' chairs and safety equipment (e.g., rescue tubes, backboards, first aid bags, breathing masks) on the pool deck are in their proper place and out of the walking paths of patrons may also reduce the possibility of injury, increase the speed and effectiveness of rescues in an emergency (Sattar, Jacobsen, Rahman, Cusack, \& Rubino, 1992), and enhance consumer satisfaction (Andaleeb, 1998). Swimming pool lifeguards are often responsible for cleaning and repositioning objects after their shifts. Because of the high turnover rate and varied work schedules among lifeguards, task expectations should be made more explicit. Task clarification, defined by Crowell, Anderson, Abel, and Sergio (1988) as the "precise specification of behavioral components
\end{abstract}


of a job" (p. 65) has been associated with behavior change when delivered in a memo and orally during a meeting (Crowell et al.).

In addition to the antecedent qualities of task clarification, consequences can be applied with the use of performance feedback (Alvero, Bucklin, \& Austin, 2001). Often, feedback is paired with task clarification both to specify and to allow the group or individuals to track their performance (Austin, Weatherly, \& Gravina, 2005; DeRiso \& Ludwig, 2007). In many published studies, behavior is monitored and feedback is given by independent observers. Self-monitoring, on the other hand, enables individuals to monitor their own performance systematically. Self-monitoring can be accomplished through the use of a behavioral checklist completed by the employee. Doll, Livesey, McHaffie, and Ludwig (2007) used self-monitoring paired with weekly graphic feedback to improve end-of-night cleaning tasks at a ski shop. Thus, self-rating of one's behaviors on a behavioral checklist can serve as a prompt (i.e., antecedent) for subsequent behaviors as well as a consequence reinforcing desired behaviors (Sulzer-Azaroff \& Mayer, 1991). The current study was designed to examine the effects of task clarification, self-monitoring, and performance feedback on the cleaning behaviors of lifeguards at a community swim complex.

\section{METHOD}

\section{Assessment}

After-hours observations at a community swim complex revealed litter and equipment left in walking paths or not replaced properly. A PIC-NIC analysis (Daniels \& Daniels, 2004) was conducted and results indicated that desired cleaning behaviors were not being properly performed due to inadequate or improper antecedents and/or a lack of consistent consequences. Lifeguards frequently began cleaning before patrons exited the building, often leading to incomplete tasks. Lifeguards were unaware of certain closing behavior standards that the managers cited. Lifeguards reported that managers delivered cleaning prompts inconsistently. Oral or written task instructions were the most conspicuous antecedents missing. Consequences for cleaning performance were inadequate or completely absent.

\section{Participants and Setting}

Lifeguards ( $n=9 ; 6$ females and 3 males) at a community swimming complex located in a small university town participated in the present study. All were college students ranging in age from 18 to 23 years $(M=20)$ with similar levels of education ( $M=3$ years of college). Employment tenure varied from 2 to 12 months. All lifeguards were paid hourly. The swim complex had an average of 50 swimmers per day during the study. During closing shifts, one or two lifeguards and one manager were on duty. End-of-shift closing tasks were to be started immediately after the swim complex closed and required approximately 30 minutes to complete properly. Nightly closing tasks were concentrated in two prominent areas, the pool deck and the lobby. All lifeguards were trained by a manager upon hiring to complete all 
targeted tasks; however, no standardized training process was in place. Also, check sheets for proper cleaning existed but were infrequently used.

\section{Dependent Measures}

Overall, 17 specific closing tasks were observed, 11 in the lobby and 6 on the pool deck. Within the lobby area, three general task clusters existed: vacuuming, garbage removal, and tidying. One specific task from each lobby cluster (listed below) was used as a control and therefore not targeted by the interventions. Specific criteria needed to be met for each lobby cleaning task to receive a "satisfactory" score. Proper vacuuming technique involved the removal of any visible debris larger than the diameter of a standard pencil eraser from the lobby and office carpeting (task 1), removable rugs (task 2), and under furniture (task 3). In addition, the vacuum was to be returned to the storage closet in the designated position with power cord wound completely around the vacuum (task 4; a control behavior not targeted by the intervention). End-of-shift garbage disposal consisted of removing and tying of all trash bags more than half full (task 5) and placing them in the foyer for pickup (task 6; control behavior). Trash cans were then to be relined with new trash bags (task 7). Equipment tidying involved the replacement of patron storage baskets on their designated shelves (task 8; control behavior), straightening of removable rugs so they lay flat and parallel to the walls (task 9), placing benches flush to designated walls (task 10), and sanitizing the stainless steel basin of the lobby water fountain so that all visible water marks were removed (task 11).

The three pool deck task clusters were as follows: deck-chair straightening, debris and lost-and-found removal, and placement of lifeguard stand items. One task from the lifeguard stand cluster (listed below) was used as a behavioral control. Task criteria for the pool deck area mandated that pool deck chairs were to be flush and centered against the support pillars around the kiddie and therapy pools (task 12) and on the north side of the lap pool (task 13). All visible debris larger than the diameter of a standard pencil eraser (task 14) and lost-and-found items (task 15) had to be removed from the pool deck area. These items were to be disposed of in the trash can or placed in the lost-and-found container located in the office. The lifeguard rescue tube was to be on the lifeguard stand platform with the strap tied up with Velcro and the "Lifeguard" text facing the pool (task 16; control behavior). Finally, both lifeguard radios were to be powered down and returned to the designated area in the office (task 17).

Data were collected 4 to 5 days per week, from October to February, except during holidays when the pool was closed. Data were collected after working hours by researchers working independently. To collect data, the authors walked separately around the lobby and pool deck thoroughly inspecting all target areas. A data collection sheet was created to record the score of all 17 tasks. Each task was rated as "satisfactory" (score $=1$ ) or "not satisfactory" (score $=0$ ) based on the task criteria described above. Interobserver reliability data were collected on approximately one-third of observation sessions across baseline, intervention, and follow-up phases. 


\section{Design}

An ABA reversal design was used in this study. After 3 weeks of baseline observations, a second 3-week phase consisted of three intervention components: task clarification, self-monitoring, and performance feedback. Control behaviors consisting of one task from each of the four cleaning clusters were not targeted by these interventions. After a hiatus of 6 weeks, follow-up observations were conducted for an additional 3 weeks after the intervention materials were removed.

Intervention Components

\section{TASK CLARIFICATION}

At the beginning of the intervention phase, all lifeguards and managers attended a half-hour task clarification meeting. A script detailing the criteria for the satisfactory completion of the targeted tasks was read and questions were answered. The script and a one-page summary were posted on the announcement board.

\section{SELF-MONITORING}

At the meeting, lifeguards and managers were instructed how to fill out the closing behavior checklist for the 13 targeted tasks based on the definitions in the task clarification script. Tasks were to be rated a "1" if the task was completed satisfactorily or if they judged the area already to be clean or tidy. Tasks they believed were not completed satisfactorily were scored as a "0." Lifeguards were not to confer with the manager during the scoring process. After lifeguards completed the closing duties, managers walked around the lobby and pool deck to complete their own completion ratings. Lifeguards were prompted to complete any unsatisfactory tasks but the "0" scores were not changed.

\section{PERFORMANCE FEEDBACK}

Graphic feedback was used to publicly display lifeguards' self-reported scores. Researcher ratings were ostensibly reported as managers' scores. In this way, lifeguards and managers were unaware that researchers were rating performance, and any bias or unreliability in managers' scores was eliminated. A time series graph was updated each night displaying the percentage of satisfactorily completed tasks.

Task clarification, self-monitoring, and performance feedback were withdrawn simultaneously after 3 weeks. A posted message announced the end of the interventions. 


\section{RESULTS}

\section{Interobserver Reliability}

Reliability data were collected on 11 of the 34 nightly observation sessions, representing $32 \%$ of all observation sessions. Overall reliability (or percentage agreement) for the baseline and intervention phases was $96.88 \%$ for ratings of vacuuming tasks, $95.83 \%$ for ratings of garbage removal, $93.75 \%$ for ratings of tidying, and $97.92 \%$ for ratings of pool deck maintenance. There were no notable differences in reliability across phases.

\section{Data Analysis}

The results represent only the data collected by the researchers and do not include lifeguard self-ratings. Nightly percent of tasks completed were averaged to yield task cluster and phase percentages. Task completion means and standard errors for each phase are presented in Table 1. The task performance by phase is aggregated across all tasks in Figure 1. Figure 2 depicts vacuuming behaviors over the course of the study; Figure 3 depicts garbage removal behaviors over the course of the study; Figure 4 depicts tidying behaviors over the course of the study; and Figure 5 depicts pool deck maintenance behaviors over the course of the study.

Despite some variation of baseline data, less than half of all targeted tasks were completed each night. Employees completed the targeted tasks more than $50 \%$ of the time in only two of the four targeted behavior areas. Task completion increased substantially: $45 \%(S E=.0013)$ during baseline to $77 \%(S E=.0007)$ during the intervention phase; a mean increase in performance of 32 percentage points ( $59 \%$ over baseline levels). A return to 
TABLE 1 Means and Standard Errors for all Tasks During Baseline and Intervention Phases

\begin{tabular}{|c|c|c|c|c|c|c|c|c|}
\hline \multirow[b]{2}{*}{ Area } & \multirow[b]{2}{*}{ Task $\#$} & \multirow[b]{2}{*}{ Task } & \multicolumn{2}{|c|}{ Baseline } & \multicolumn{2}{|c|}{ Intervention } & \multicolumn{2}{|c|}{ Follow-up } \\
\hline & & & $M$ & $S E$ & $M$ & $S E$ & M & $S E$ \\
\hline \multirow[t]{20}{*}{ Lobby } & & Vacuuming $(\%)$ & & & & & & \\
\hline & 1 & General vacuuming & $13.3 \%$ & 0.091 & $61.9 \%$ & 0.112 & $28.6 \%$ & 0.125 \\
\hline & 2 & Removable rugs & $20.0 \%$ & 0.107 & $47.6 \%$ & 0.114 & $35.7 \%$ & 0.133 \\
\hline & 3 & Under fumiture & $0.0 \%$ & 0.000 & $52.4 \%$ & 0.114 & $0.0 \%$ & 0.000 \\
\hline & $4 \mathrm{c}$ & Vacuum in closet & $33.3 \%$ & 0.126 & $85.7 \%$ & 0.082 & $92.9 \%$ & 0.071 \\
\hline & & $\begin{array}{l}\text { Overall vacuuming } \\
\text { (Excluding control) }\end{array}$ & $11.1 \%$ & 0.002 & $54.0 \%$ & 0.004 & $21.4 \%$ & 0.064 \\
\hline & & Garbage remoual $(\%)$ & & & & & & \\
\hline & 5 & Removal of bags & $66.7 \%$ & 0.126 & $100.0 \%$ & 0.000 & $71.4 \%$ & 0.125 \\
\hline & $6 \mathrm{c}$ & Placement in foyer & $46.7 \%$ & 0.133 & $76.2 \%$ & 0.099 & $71.4 \%$ & 0.125 \\
\hline & 7 & Relining of trash cans & $80.0 \%$ & 0.107 & $100.0 \%$ & 0.000 & $92.9 \%$ & 0.071 \\
\hline & & $\begin{array}{l}\text { Overall garbage removal } \\
\text { (excluding control) }\end{array}$ & $73.3 \%$ & 0.007 & $100.0 \%$ & 0.000 & $82.1 \%$ & 0.074 \\
\hline & & Tidying $(\%)$ & & & & & & \\
\hline & $8 \mathrm{c}$ & Storage baskets & $80.0 \%$ & 0.107 & $71.4 \%$ & 0.105 & $92.9 \%$ & 0.071 \\
\hline & 9 & Removable rugs & $26.7 \%$ & 0.118 & $90.5 \%$ & 0.069 & $21.4 \%$ & 0.114 \\
\hline & 10 & Lobby benches & $53.3 \%$ & 0.133 & $76.2 \%$ & 0.099 & $14.3 \%$ & 0.097 \\
\hline & 11 & Water fountain & $20.0 \%$ & 0.107 & $71.4 \%$ & 0.099 & $7.1 \%$ & 0.071 \\
\hline & & Overall tidying & $33.3 \%$ & 0.005 & $79.4 \%$ & 0.003 & $14.3 \%$ & 0.055 \\
\hline & & Pool deck maintenance & $\%$ & & & & & \\
\hline & 12 & Chair position on beam & $33.3 \%$ & 0.126 & $90.5 \%$ & 0.069 & $35.7 \%$ & 0.133 \\
\hline & 13 & Chair location on deck & $93.3 \%$ & 0.067 & $100.0 \%$ & 0.000 & $85.7 \%$ & 0.970 \\
\hline \multirow[t]{7}{*}{ Pool deck } & 14 & Debris removed & $6.7 \%$ & 0.067 & $33.3 \%$ & 0.109 & $7.1 \%$ & 0.071 \\
\hline & 15 & Lost/found removed & $80.0 \%$ & 0.107 & $85.7 \%$ & 0.082 & $92.9 \%$ & 0.071 \\
\hline & $16 \mathrm{c}$ & Rescue tube position & $40.0 \%$ & 0.131 & $19.0 \%$ & 0.092 & $35.7 \%$ & 0.133 \\
\hline & 17 & Radio placement & $93.3 \%$ & 0.067 & $90.5 \%$ & 0.069 & $92.9 \%$ & 0.071 \\
\hline & & $\begin{array}{l}\text { Overall pool deck } \\
\text { maintenance }\end{array}$ & $61.3 \%$ & 0.003 & $80.0 \%$ & 0.002 & $62.9 \%$ & 0.058 \\
\hline & & Overall $(\%)$ & $45.1 \%$ & 0.001 & $76.9 \%$ & 0.001 & $45.1 \%$ & .037 \\
\hline & & $\begin{array}{l}\text { Overall }(\%) \text { control } \\
\text { behaviors }\end{array}$ & $50.0 \%$ & .064 & $63.1 \%$ & .053 & $73.2 \%$ & .060 \\
\hline
\end{tabular}

$c-$ control

baseline was observed once the intervention ceased and the follow-up phase was initiated. Overall performance decreased to $45 \%(S E=.03698)$ of tasks completed during the follow-up phase.

Of the four nontargeted control tasks, two tasks (position of the vacuum and placement of full trash bags) increased when the intervention was implemented. The other two control tasks (return of storage and position of the rescue tube) decreased during this study .

\section{Accuracy of Self-Monitoring and Manager Monitoring}

In order to measure the reliability of self-monitoring across lifeguards, managers and researchers, we conducted Pearson correlations on the data collected 


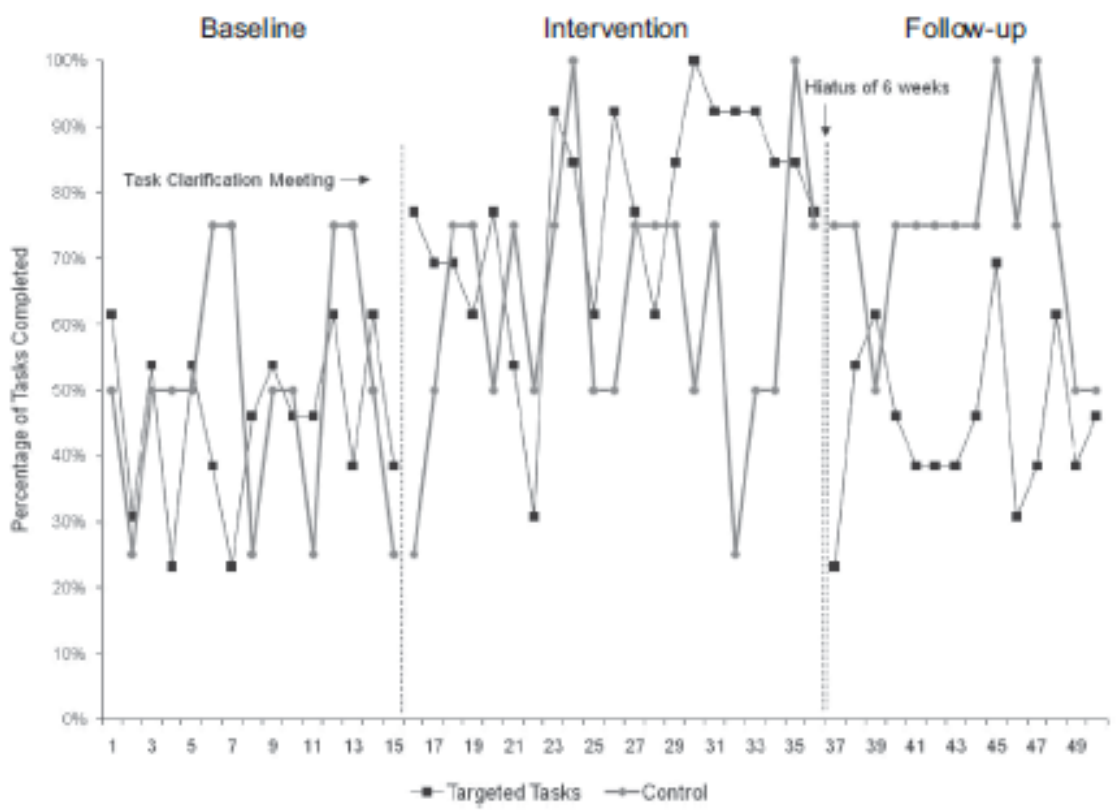

FIGURE 1 Overall task completion, including control, across baseline, intervention, and follow-up phases Each data point on the overall results graph represents one observation session.

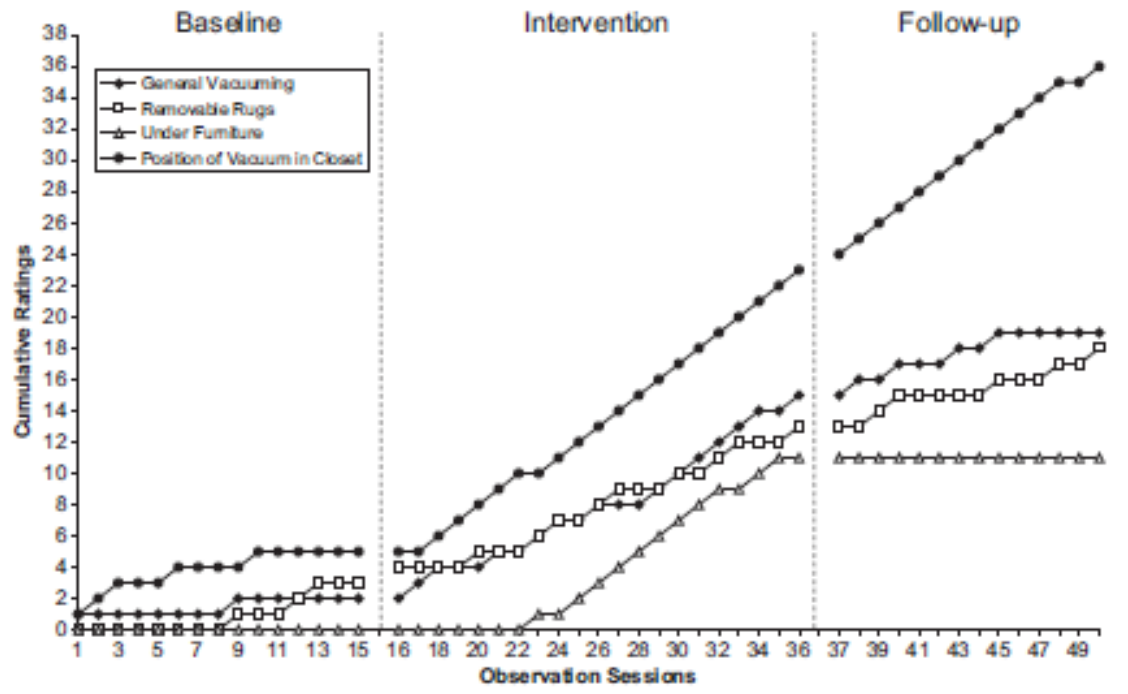

FIGURE 2 Cumulative ratings of task completion in the Vacuuming Cluster.

by these groups. Managers' estimates of task completion correlated at $r=.46$ with the researchers' data. Employee estimates of their own task completion correlated at $r=.36$ with the researchers'. Managers' and employees' estimates of correct performance only correlated at $r=.17$. 


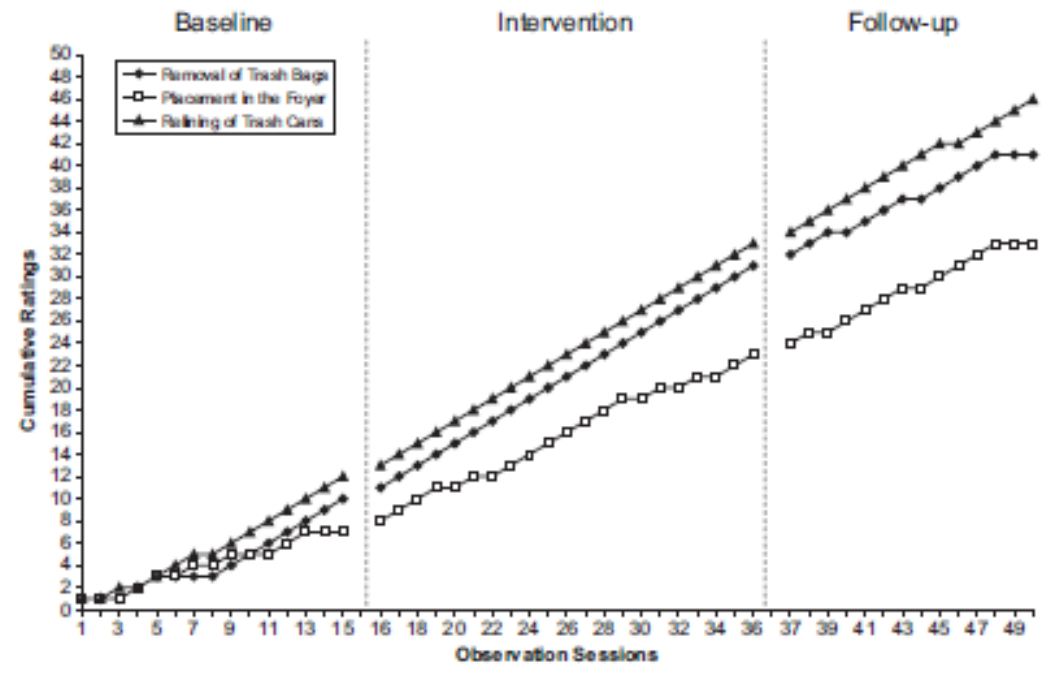

FIGURE 3 Cumulative ratings of task completion in the Garbage Removal Cluster.

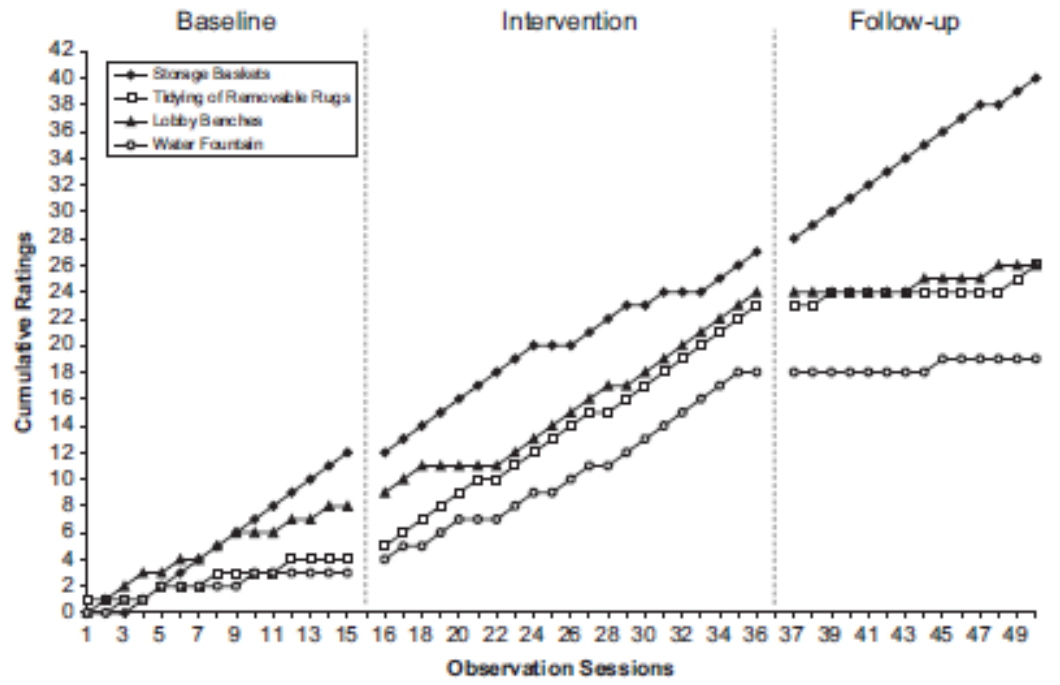

FIGURE 4 Cumulative ratings of task completion in the Tidying Cluster.

\section{DISCUSSION}

These findings are consistent with the experimental literature suggesting that task clarification, self-monitoring, and graphic feedback help increase desired performance. Performance improved gradually during the intervention most likely due to employees becoming more familiar with the checklist used 


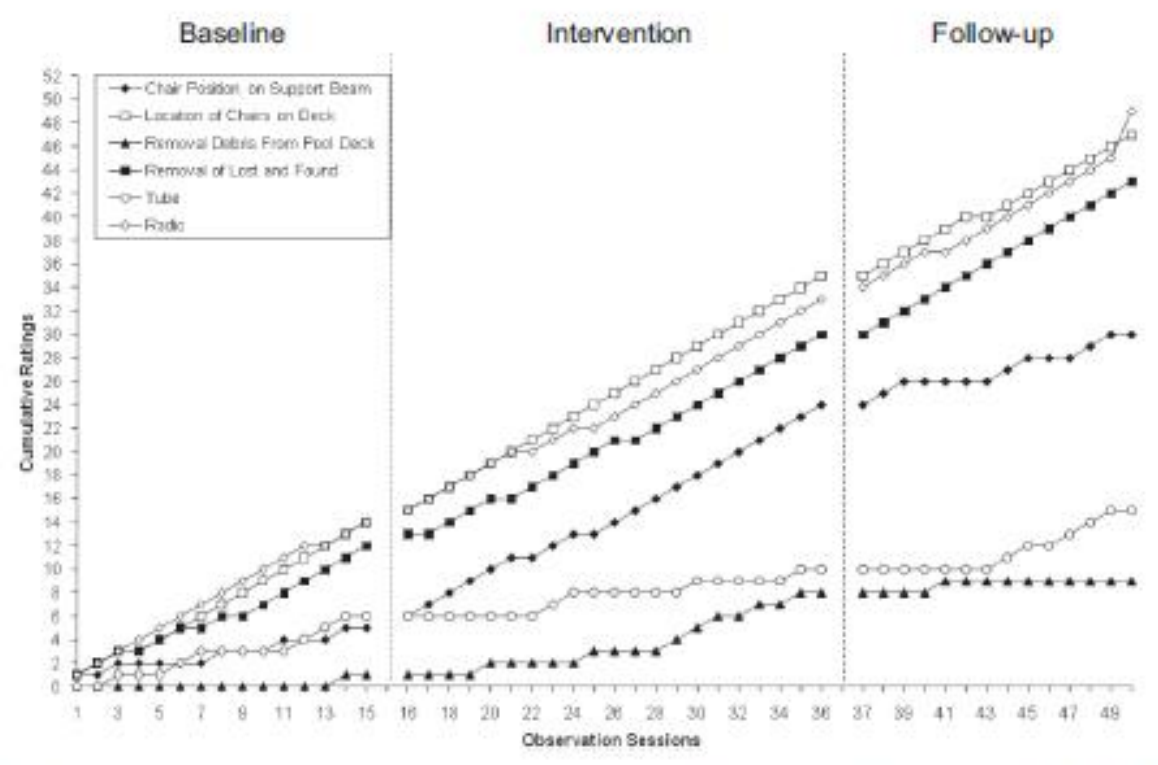

FIGURE 5 Cumulative ratings of task completion in the Pool Deck Maintenance Cluster Each data point on the overall results graph represents one observation session.

each night, which, along with feedback, eventually shaped the numerous desirable behaviors, Thus, the discriminate antecedent qualities of the checklist also became stronger over time. It is also possible that the employee had to reduce normal closing behaviors (e.g., talking with one another) prior to developing a new routine of behaviors to accommodate the checklist. The most substantial improvements in task completion were seen in clearly defined tasks such as vacuuming. The smallest improvements were observed in tasks covering a large area (e.g., pool deck maintenance or tasks already completed at a high rate during baseline (e.g., deck chair placement, lost and found removal, lifeguard radio placement).

Self-monitoring may be a particularly useful intervention to influence lifeguards' closing cleaning behaviors. The self-monitoring checklist instructed employees as to what needed to be completed before closing. If employees had further questions they could consult the task clarification script. Further, the graphic feedback allowed employees to evaluate their performance compared to that of their coworkers. This may have created an opportunity for discussions of cleaning among the employees and between employees and managers.

The Pearson Correlation of lifeguard, manager, and researcher data suggested that the three groups did not reliably agree on the definition of correct nightly cleaning performance and did not have a common understanding of correct performance. Managers seemed more able to estimate correct performance than lifeguards. Knowing this, a more interactive training task clarification program could be implemented to increase the common understanding of correct cleaning performance.

The graphic feedback presented employees a comparison between their 
actual level of performance vs. their self-monitored level of performance. This may have helped to improve their self-monitoring skills. Perhaps a longer intervention period is warranted for employees to learn how to selfmonitor performance more accurately.

Nontargeted behaviors making up the control tasks also changed during the intervention and follow-up period although not systematically. Additionally, performance in all control tasks increased after the intervention phase ended. It is possible that there were unpredictable relationships among the targeted and control tasks that led to changes in control tasks. Control tasks that decreased in performance were likely the result of reinforcing competing behaviors. Also, there may have been a greater opportunity to perform the control task because of proximity to targeted tasks.

\section{Limitations and Future Studies}

The demonstration of experimental control (Kazdin, 1994) was limited. A randomly assigned control group was not possible due to the small number of lifeguards and scheduling complexities. The number of patrons visiting the swim complex decreased across the study according to seasonal patterns, which may have led to a cleaner facility and a decreased need for cleaning. Data collectors were not blind to experimental phases, possibly biasing ratings of lifeguard performance despite efforts to maximize interobserver reliability. As task observations were conducted after closing, actual lifeguard behaviors could not be observed. Although rating lifeguards after closing eliminated most observer effects, it was possible that the lifeguards did not complete a task because it was already clean or tidy from previous cleanings. Future studies would benefit from staggering components of an intervention in a multiple baseline to investigate any differential effects of task clarification, self-monitoring, and feedback on cleaning behavior. Along with self-monitoring and performance feedback, employees should have the opportunity to create goals for themselves, which Kazdin (1974) suggested will improve performance in the context of self-monitoring.

An intervention that includes techniques such as task clarification, selfmonitoring, and performance feedback has little cost when compared to the benefits it provides an organization. Improving the cleanliness of businesses such as swimming complexes could possibly bring in new members, improve retention, and thereby increase monetary income. Cleanliness interventions could also keep the patrons from getting sick or injured, which keeps the business from being held liable for such incidents, likewise, self-monitoring, unlike many other intervention techniques, is a technique that can be institutionalized by the organization. It also requires little training and, once checklists have been created, no additional intervention materials are needed. Organizations with a large employee base or multiple shifts may also benefit from intervention packages such as this one because it can reach and help manage a wide selection of employees. Organizations whose working environments are often changing can use the task clarification meetings to their advantage to help maintain correct performance. 


\section{REFERENCES}

Alvero, A. M., Bucklin, B. R., \& Austin, J. (2001). An objective review of the effectiveness and essential characteristics of performance feedback in organizational settings. Journal of Organizational Behavior Management, 21, 3-30.

Andaleeb, S. S. (1998). Determinants of customer satisfaction with hospitals: A managerial model. International Journal of Health Care Quality Assurance, 11, 181-187.

Austin, J., Weatherly, N. L., \& Gravina, N. E. (2005). Using task clarification, graphic feedback, and verbal feedback to increase closing-task completion in a privately owned restaurant. Journal of Applied Behavior Analysis, 38, 117-120.

Crowell, C. R., Anderson, C. D., Abel, D. M., \& Sergio, J. P. (1988). Task clarification, performance feedback, and social praise: Procedures for improving the customer service of bank tellers. Journal of Applied Behavior Analysis, 21, 65-71.

Daniels, A. C., \& Daniels, J. E. (2004). Performance management: Changing behavior that drives organizational effectiveness (4th ed., Rev. ed.). Performance Management. Atlanta, GA: Aubrey Daniels International.

DeRiso, A., \& Ludwig, T. D. (2007). Task clarification of cleaning and restocking behaviors for a fine dining restaurant. Manuscript submitted for publication.

Kazdin, A. E. (1974). Reactive self-monitoring: The effects of response desirability, goal setting, and feedback. Journal of Consulting and Clinical Psychology, 42, 704-716.

Kazdin, A. E. (1994). Behavior modification in applied settings. Pacific Grove, CA: Brooks/Cole.

Sattar, S. A., Jacobsen, H., Rahman, H., Cusack, T. M., \& Rubino, J. R. (1994). Interruption of rotavirus spread through chemical disinfection. Infection Control and Hospital Epidemiology, 15, 751-756.

Sulzer-Azaroff, B., \& Mayer, G. R. (1991). Behavior analysis for lasting change. Fort Worth, TX: Holt, Rinehart, and Winston. 